\title{
Interférences
}

Ars scribendi

11 | 2018

Poésie latine et miroir du prince

\section{Phoébus et Phaéthon dans les Métamorphoses : la mythologie ovidienne comme miroir du Prince inversé}

\section{Aliénor Cartoux}

\section{(2) OpenEdition \\ Journals}

Édition électronique

URL : https://journals.openedition.org/interferences/6395

DOI : 10.4000/interferences.6395

ISSN : $1777-5485$

Éditeur

HiSoMA - Histoire et sources des Mondes antiques

\section{Référence électronique}

Aliénor Cartoux, «Phoébus et Phaéthon dans les Métamorphoses : la mythologie ovidienne comme miroir du Prince inversé », Interférences [En ligne], 11 | 2018, mis en ligne le 26 juillet 2018, consulté le 21 septembre 2021. URL : http://journals.openedition.org/interferences/6395 ; DOI : https://doi.org/ 10.4000/interferences.6395

Ce document a été généré automatiquement le 21 septembre 2021.

Tous droits réservés 


\title{
Phoébus et Phaéthon dans les Métamorphoses : la mythologie ovidienne comme miroir du Prince inversé
}

\author{
Aliénor Cartoux
}

$1 \mathrm{Si}$, comme nous l'avons vu dans l'Introduction de ce numéro, le genre du miroir du Prince n'est réellement apparu qu'au cours du Moyen-Âge, le corpus que nous souhaitons analyser a ceci de particulier qu'il est le seul des textes ici étudiés à être antérieur à la réflexion fondatrice du De Clementia de Sénèque, introduisant la métaphore catroptique ${ }^{1}$. Pour autant, de même que les codifications théoriques d'un genre ne sont pas nécessaires à son apparition, de facto dans la littérature antérieure, dont on théorise ensuite les invariants de manière empirique, de même il n'est pas nécessairement besoin qu'un auteur politico-philosophique ait mis en mots cette spécificité de la littérature pour que d'autres auteurs, avant lui, en aient ressenti le pouvoir, consciemment ou non ${ }^{2}$.

2 Interroger le mythe de Phaéthon dans les Métamorphoses par le prisme du miroir du Prince n'est donc pas, a priori, une entreprise totalement insensée ${ }^{3}$. Pourtant, à la lecture du mythe lui-même ${ }^{4}$, il semble bien que le concept de miroir du Prince soit inopérant pour éclaircir notre compréhension du texte: à première vue, celui-ci échappe aussi bien à la forme qu'au contenu requis pour pouvoir être désigné ainsi. Tout d'abord, parce qu'il s'agit d'une œuvre mythologique et donc, par définition, empreinte, sinon de fausseté, du moins d'une incertitude insurmontable quant à la véracité de son contenu ${ }^{5}$; de même, la lettre du mythe est, de manière inhérente à sa nature, coupée de l'actualité politique contemporaine de l'auteur, et est donc peu propice à contenir des conseils au Prince qui lui seraient directement adressés. Enfin, la spécificité du mythe de Phaéthon est que, à la différence de bien des mythes des Métamorphoses, le narrateur se garde bien ici d'en proposer une quelconque analyse morale et éthique ${ }^{6}$ : dès lors, la dimension didactique fondamentale à tout miroir du 
Prince ne peut être assumée. Enfin, l'histoire qui nous occupe est, littéralement, une histoire de reconnaissance entre un père et son fils, elle est donc bien peu adaptée à recevoir les conseils d'un poète à son dirigeant.

3 La tâche que nous nous sommes donnée est donc osée, et sans doute l'angle sous lequel nous allons aborder le mythe de Phaéthon n'était-il pas consciemment suggéré par Ovide. Nous voudrions cependant montrer que le concept de miroir du Prince, pour anachronique qu'il puisse être, permet de révéler plus distinctement la lecture allégorique et les enjeux politiques de ce mythe qui, eux assurément, étaient bien voulus par l'auteur.

Dès lors que l'on interroge le mythe de Phaéthon avec les éléments d'analyse du miroir du Prince, un passage en particulier semble correspondre à la définition qu'on reconnaît à cette notion ${ }^{7}$ : les deux discours que Phoébus tient à son fils, l'un pour le dissuader de demander à conduire le char solaire ${ }^{8}$, l'autre pour lui indiquer, une fois le premier avertissement méprisé par le jeune homme, comment le conduire ${ }^{9}$. Ces discours sont tous deux qualifiés de monitus ${ }^{10}$, la première fois par le narrateur, qui clôt ainsi la parole du Soleil ${ }^{11}$, la seconde fois par Phoébus lui-même, qui introduit ainsi son propre discours et le termine en donnant à Phaéthon ce qu'il qualifie de derniers consilia $^{12}$. La portée didactique des propos du Soleil est donc mise en valeur, d'abord par le narrateur, à destination du lecteur, puis, au sein de la fiction diégétique elle-même, à destination du personnage fictif de Phaéthon. À l'intérieur de ces discours, on retrouve certaines des modalités énonciatives recommandées par la portée didactique du miroir (Ov., Met. 2, 53-56) :

Dissuadere licet; non est tua tuta uoluntas ;

Magna petis, Phaethon, et quae nec uiribus istis

Munera conueniant nec tam puerilibus annis.

Sors tua mortalis; non est mortale quod optas.

«Je peux tenter de te dissuader; ce que tu veux n'est pas sans danger. C'est une grande faveur que tu demandes, Phaéthon, et qui ne convient ni à tes forces, ni à tes jeunes années. Ton sort est celui d'un mortel; ce n'est pas l'affaire d'un mortel ce que tu souhaites » [trad. J. Chamonard modifiée $]^{13}$.

5 La portée didactique du discours du Soleil est ici mise en valeur par l'allure sentencieuse des phrases, tout d'abord grâce au mélange opéré entre présent d'énonciation et présent de vérité générale. Le début du discours est particulièrement représentatif de ce procédé, puisque la brièveté de l'expression Dissuadere licet permet d'y voir à la fois une réflexion d'ordre général, mais aussi une affirmation en contexte, où le pronom personnel me serait sous-entendu. C'est donc également la brièveté des formules qui donne aux propositions du dieu la solennité caractéristique de toute maxime. Enfin, on trouve aussi, dans les deux discours du Soleil, de très nombreux impératifs qui assurent la modalité injonctive nécessaire au miroir du Prince ${ }^{14}$.

On peut alors se demander pourquoi les discours de Phoébus à Phaéthon, c'est-à-dire d'un père à son fils, semblent si bien correspondre à un miroir du Prince. C'est que, comme l'ont déjà relevé d'autres critiques ${ }^{15}$, bien que dans une optique différente, le mythe de Phaéthon ne peut acquérir toute son épaisseur que si l'on accepte d'en faire une lecture allégorique concernant la gestion d'un pouvoir de type monarchique. De fait, Ovide emploie à dessein plusieurs mots ou expressions dont la polysémie permet de superposer un sens figuré, entièrement politique, au sens premier et explicite. C'est ce procédé métaphorique que le poète fait utiliser à Phoébus lui-même lorsqu'il adresse ses conseils à son fils (Ov., Met. 2, 84-86) : 
Nec tibi quadrupedes animosos ignibus illis

Quos in pectore habent, quos ore et naribus efflant,

In promptu regere est ; [...]

"Quant aux chevaux, animés par le feu qui brûle dans leur poitrail et qu'ils soufflent par la bouche et les naseaux, les conduire ne saurait être pour toi entreprise facile $»$.

7 La conduite des chevaux solaires est comparée ici à la direction d'un gouvernement : le verbe à l'infinitif, regere, permet la superposition des sens, en sorte que les chevaux semblent représenter le peuple qui, lorsqu'il est livré à sa propre fureur, au cours d'une sédition, n'est que difficilement contrôlable. Or, et cela conforte notre interprétation, le narrateur joue de la métaphore utilisée par son personnage pour la filer implicitement tout au long de son récit, mais en l'inversant (Ov., Met. 2, 150-152) :

Occupat ille leuem iunenali corpore currum

Statque super manibusque leues contingere habenas

Gaudet et inuito grates agit inde parenti.

«Mais Phaéthon prend possession du char bien léger sous son jeune corps; il s'y

dresse, se réjouit de toucher de ses mains les rênes légères, et ensuite remercie son père qui a agi bien malgré lui » [trad. J. Chamonard modifiée].

Dans cette prise de parole du narrateur, ce n'est plus le lexique politique qui s'immisce dans celui de l'aurige mais bien le vocabulaire équestre qui doit être analysé dans une perspective de gouvernement politique. Cette lecture est facilitée par l'analogie fréquente qui est faite chez les auteurs latins entre gouvernant et capitaine de navire ou conducteur de char ${ }^{16}$. Enfin, toute l'isotopie du poids, qu'Ovide s'amuse à développer par la suite ${ }^{17}$, et dont la polysémie est très fréquemment employée, même dans le vocabulaire courant, dans son acception politique ${ }^{18}$, conforte l'idée de la nécessité de faire une lecture allégorique de ce mythe.

9 Dans cette perspective, Phoébus et Phaéthon ne doivent plus être considérés simplement comme un père face à son fils, mais bien comme représentants paradigmatiques, typiques des exempla déployés dans les miroirs du Prince. Le Soleil, tout d'abord, est l'emblème du roi idéal: les premiers mots du mythe désignent son palais comme royal ${ }^{19}$, et les battants de ses portes extérieures soulignent qu'il n'est pas roi d'un simple pays, mais du monde tout entier ${ }^{20}$. La magnificence même de son palais, hyperbolique en ce qu'elle fusionne tous les matériaux les plus précieux aux yeux des Romains ${ }^{21}$, témoigne de l'importance du personnage qu'il abrite. La première apparition du Soleil est également celle d'un roi dont l'autorité n'est nullement contestée (Ov., Met. 2, 25-30) :
A dextra laeuaque Dies et Mensis et Annus
Saeculaque et positae spatiis aequalibus Horae
Verque nouum stabat cinctum florente corona,
Stabat nuda Aestas et spicea serta gerebat,
Stabat et Autumnus, calcatis sordidus uuis,
Et glacialis Hiems, canos hirsuta capillos.
«À droite et à gauche, étaient le Jour, le Mois, l'Année, les Siècles et, placées à des intervalles égaux, les Heures. Là se tenait aussi le Printemps renouvelé, la tête ceinte d'une couronne de fleurs; là se tenait l'Été, nu, qui portait une guirlande d'épis; là se tenait l'Automne barbouillé du jus des grappes foulées, et l'Hiver de glace, à la chevelure blanche en désordre » [trad. J. Chamonard modifiée].

Les indications spatiales du passage (a dextra leuaque; spatiis aequalibus) soulignent l'impression que doit ressentir le lecteur, celle d'un royaume parfaitement en ordre, que rend la structure des vers ${ }^{22}$. Les personnifications des diverses étapes de l'année, 
qui dépendent toutes de l'évolution de la course du Soleil, peuvent bien être comprises comme les représentantes allégoriques des différentes couches de la nobilitas qui ne peuvent bénéficier de leur juste fonctionnement que grâce à la présence irradiante de leur monarque, autour de qui l'ordre peut se créer. Ainsi, lorsque le Soleil affirme à Phaéthon (Ov., Met. 2, 98-99) :

Deprecor hoc unum, quod uero nomine poena,

Non honor est ; poenam, Phaethon, pro munere poscis.

"Je ne te supplie de renoncer qu'à cette seule chose, dont le vrai nom est châtiment, et non honneur. C'est un châtiment, Phaéthon, que tu demandes comme une faveur.»

il s'agit de faire du dieu l'incarnation du bon roi, celui dont la sagesse est un gage de bon gouvernement. Sa réflexion est en effet proche de la définition du philosophe-roi de Platon ${ }^{23}$, qui dirige non pas par orgueil mais par devoir, parce que lui seul est apte à remplir cette fonction ${ }^{24}$.

La fonction didactique du mythe est soulignée par la composition dichotomique des personnages : à la sagesse du roi s'oppose la fougue inexpérimentée ${ }^{25}$ de l'apprenti-roi qu'est Phaéthon. Son ignorance ${ }^{26}$ ainsi que sa jeunesse ${ }^{27}$ sont soulignées de nombreuses fois ; sa qualification de puer est à ce titre particulièrement révélatrice ${ }^{28}$, car, bien que le terme, dans la bouche de Phoébus, ait évidemment le sens de «mon fils », il semble que l'acception «jeune enfant» s'y superpose et légitime les conseils que le Soleil donne à un fils encore inexpérimenté. Phaéthon est donc le modèle parfait du Prince dont l'instruction doit être faite : son ascendance royale a été réaffirmée par son géniteur lui-même au début du livre ${ }^{29}$, et, de ce fait, il peut prétendre à régner un jour sur le domaine paternel; mais, dans la mesure où il rencontre son père pour la première fois $^{30}$, toute son éducation de Prince divin reste à faire.

12 L'histoire de Phoébus et de Phaéthon se présente bien avec les caractéristiques inhérentes au miroir du Prince. Ce qui, de prime abord, nous semblait contraire à une telle définition, à savoir un mythe atemporel, coupé de la réalité immédiate, est en fait un habile procédé qui permet de réaliser un miroir du Prince indirect, parce qu'allégorique $^{31}$ : le mythe agit ici comme le songe dans certains miroirs de l'époque médiévale ${ }^{32}$. Or, pour Ovide, ce procédé de détournement présente plusieurs avantages compte tenu de la réalité politique du temps : Apollon, dont la caractérisation solaire est propre à l'époque augustéenne ${ }^{33}$, fait office de figure médiane entre le poète et le Prince. Seul le dieu protecteur d'Auguste, en effet, est apte à lui prodiguer des conseils. Mais, patron divin des poètes, il est également capable de se faire le porte-parole d'Ovide qui, ainsi, n'a pas l'indécence de suggérer que lui, simple poète, peut expliquer au Prince, au pouvoir depuis longtemps déjà, comment gouverner. Le personnage de Phaéthon a bien la même fonction : les conseils du mythe sont, stricto sensu, adressés uniquement à un personnage intradiégétique, dont on a eu soin de mentionner, avant même le début du livre 2, la jactance et l'orgueil ${ }^{34}$. Le leitmotiv de sa jeunesse est également le medium par lequel le miroir du Prince peut éviter l'irrévérence. Certes, ce dernier point contrevient au miroir indirect du Prince, qui voudrait que le représentant mythologique soit identique à la personnalité réelle qu'elle supplée ${ }^{35}$. Mais la jeunesse de l'apprenti-roi est un élément fondamental dans tout miroir pour justifier les conseils prodigués, et, sur certains points essentiels, Phaéthon nous semble bien être le représentant d'Auguste : le lien personnel que tous deux revendiquent avec la figure de Phoébus est au centre de leurs prétentions à gouverner ${ }^{36}$; mais le parallèle le plus fort est leur nature profondément ambiguë, ni tout à fait humaine, ni tout à fait divine, et 
leur volonté, de ce fait, de devenir dieux. Cette prétention est, dans ce mythe, traduite par la représentation concrète et insistante d'une apothéose mythologique (Ov., Met. 1, 776-777):

Emicat extemplo laetus post talia matris

Dicta suae Phaethon et concipit aethera mente

« Aussitôt, tout joyeux, quand sa mère lui eut ainsi parlé, Phaéthon bondit et, dans

son esprit, il convoite le ciel » [trad. J. Chamonard modifiée].

Ici, le mythe commence par retranscrire la première phase des prétentions divines du jeune homme, qui n'en sont qu'au stade du dessein, lorsque Phaéthon prend connaissance de son ascendance divine, qu'il juge encore hypothétique. Mais les desseins humains se traduisent progressivement en action concrète, lorsque Phaéthon parvient à conduire le char du Soleil à travers le ciel, malgré les avertissements de son divin père (Ov., Met. 2, 187-188) :

Quid faciat? multum caeli post terga relictum,

Ante oculos plus est; animo metitur utrumque

«Que faire? Il a, derrière son dos, laissé un vaste espace de ciel; devant ses yeux, il

y en a plus encore ; par la pensée, il mesure les deux distances ».

Dans la perspective d'une lecture allégorique, la narration retranscrirait ici la deuxième phase du processus de la divinisation de Phaéthon, dans une vision qui est horizontale, contrairement au mouvement vertical que laisserait attendre la montée vers les cieux. Le personnage se trouve alors au stade de la réalisation, bien que douloureuse. Dans les cieux, sur le char solaire, Phaéthon est ici à mi-chemin entre humanité et divinité, ne pouvant ni revenir à l'une, ni atteindre la seconde. Enfin, la dernière étape du mythe retrouve la ligne verticale qui convient au phénomène de l'apothéose, mais là encore, dans un mouvement qui s'oppose à celui attendu par le lecteur (Ov., Met. 2, 319-322) :

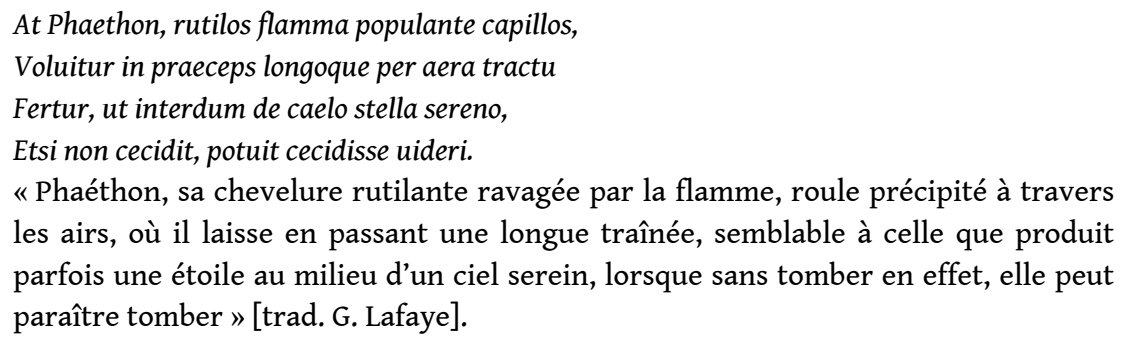

Le phénomène ici décrit rappelle sans équivoque, pour le lecteur contemporain, le catastérisme de César ${ }^{37}$, sur lequel Octavien puis Auguste fonde ses propres prétentions divines (voir Ov., Met. 15). Or, il n'est pas sans intérêt de remarquer qu'Ovide souligne dans ces vers la fausse impression d'un catastérisme qui redescendrait paradoxalement sur terre : il s'agit peut-être de souligner que ce qui pourrait sembler être un échec de Phaéthon est en réalité un accomplissement, bien qu'il se fasse à ses dépens.

Ainsi, le mythe de Phaéthon agit comme un miroir du Prince indirect, qui alerterait Auguste sur les dangers que ses prétentions à la divinisation de plus en plus patentes ${ }^{38}$ lui feraient encourir personnellement (puisque, pour devenir dieu, il faut d'abord mourir ${ }^{39}$ ) mais qui menaceraient aussi l'Empire tout entier ${ }^{40}$ (puisque celui-ci manquerait alors de l'appui solide d'un chef fort, et risquerait de sombrer dans des guerres civiles de succession).

Pourtant, un certain nombre d'éléments essentiels du miroir du Prince sont ici absents ou bien même mis en défaut : tout d'abord, il convient de remarquer que, malgré la forme on ne peut plus didactique des conseils du Soleil à son fils, la leçon échoue. 
Certes, cela peut s'expliquer par le rôle de contre-modèle qui est conféré à Phaéthon : Ovide donne ainsi à voir les risques auxquels on s'expose si l'on ne suit pas les conseils avisés de Phoébus. Mais l'absence de morale explicite assumée par la voix poétique achève de brouiller l'interprétation de ce mythe dont la fin est d'ailleurs ambiguë : tandis que la voix poétique reste purement narrative, la seule interprétation que l'on nous donne de la mort de Phaéthon est une épitaphe qui loue le courage du jeune homme ${ }^{41}$. La narration s'achève, en outre, par la mise en valeur du deuil familial que cette mort provoque. Comme l'ont fait remarquer certains critiques $^{42}$, Ovide prend ses distances par rapport à la tradition hellénique du mythe, en focalisant la fin de sa narration sur les conséquences humaines de la catastrophe, le deuil et la souffrance. Que faut-il conclure? Que Phaéthon a eu tort, ou bien qu'il fut le jouet de forces divines qui le dépassaient, et qu'il en fut puni bien durement par Jupiter? Enfin, c'est la portée même des conseils que le Soleil donne à son fils qui contrevient au miroir du Prince : ils ne sont pas d'ordre éthique, visant à faire de Phaéthon un bon aurige pour l'univers, mais purement pragmatiques, puisque Phoébus cherche simplement à éviter la mort du jeune homme $e^{43}$. Plus encore, ce qui le motive à vouloir éviter la mort de Phaéthon, ce n'est pas la perspective du salut commun, mais bien une considération toute personnelle, l'amour qu'il éprouve pour son fils ${ }^{44}$. Phoébus se trouve alors bien loin du modèle idéal nécessaire pour dispenser des conseils salutaires concernant l'univers.

C'est que, selon nous, le Soleil n'est pas, ou pas uniquement, le porte-parole du poète, qui expliquerait à Auguste comment diriger, alors que, au pouvoir depuis longtemps déjà, il a pu démontrer la stabilité de son Principat. Lorsque l'on étudie les caractéristiques propres au personnage de Phoébus, on constate en effet que les connotations augustéennes y sont très présentes. Il apparaît dès lors bien plus comme le dieu protecteur d'Auguste que comme le patron des poètes ${ }^{45}$. Tout d'abord, la dimension solaire d'Apollon est une spécificité de l'époque augustéenne, en accord avec la propagande du Prince ${ }^{46}$. Ensuite, la description que fait le poète du palais du Soleil rappelle par bien des aspects le temple d'Apollon Palatin (Ov., Met. 2, 1-18) ${ }^{47}$ :

Regia Solis erat sublimibus alta columnis,

Clara micante auro flammasque imitante pyropo ;

Cuius ebur nitidum fastigia summa tegebat,

Argenti bifores radiabant lumine ualuae.

Materiam superabat opus; nam Mulciber illic

Aequora caelarat medias cingentia terras

Terrarumque orbem caelumque, quod imminet orbi.

Caeruleos habet unda deos, Tritona canorum

Proteaque ambiguum ballenarumque prementem

Aegaeona suis immania terga lacertis

Doridaque et natas, quarum pars nare uidetur,

Pars in mole sedens uiridis siccare capillos,

Pisce uehi quaedam; facies non omnibus una,

Non diuersa tamen, qualem decet esse sororum.

Terra uiros urbesque gerit siluasque ferasque

Fluminaque et nymphas et cetera numina ruris.

Haec super imposita est caeli fulgentis imago

Signaque sex foribus dextris totidemque sinistris.

«Le palais du Soleil s'élevait porté par de hautes colonnes, tout brillant du scintillement de l'or et du pyrope imitant les flammes; l'ivoire luisant en recouvrait, tout en haut, le faite; les doubles battants des portes rayonnaient de l'éclat de l'argent. Mais l'art l'emportait encore sur la matière. Car Mulciber y avait ciselé les eaux entourant les terres placées au milieu, l'orbe du monde et le ciel, 
suspendu au-dessus du monde. L'onde a ses dieux, de la couleur des flots : le sonore Triton, Protée aux formes changeantes, et Aegeon s'appuyant de ses bras sur l'énorme échine des baleines, Doris et ses filles, dont on voit les unes nager, d'autres, assises sur un enrochement, sécher leur verte chevelure, certaines se faire porter à dos de poisson. Elles n'ont pas toutes même visage, sans être cependant dissemblables, ainsi qu'il convient à des sœurs. La terre porte des hommes et des villes, des forêts et des bêtes, des fleuves et des nymphes et toutes les autres divinités rustiques. Au-dessus de ces représentations est placée l'image du ciel resplendissant, et les signes du zodiaque, six sur le battant de droite, autant sur celui de gauche » [trad. J. Chamonard modifiée].

Dès le premier vers, l'élévation du palais du Soleil rappelle la position dominante du temple d'Apollon Palatin, en haut de la colline qui surplombe Rome tout entière. Si les matériaux mentionnés traduisent le raffinement de l'architecture augustéenne, la mention de l'or, que l'on retrouve également en bonne place dans la description du char solaire ${ }^{48}$, peut également être allégorique du saeculum aureum que le Principat prétend avoir instauré ${ }^{49}$. La mise en place ordonnée des signes du zodiaque rappelle le rôle qu'a joué Auguste dans la reconstitution du temps et du calendrier civique, et l'importance qu'il a accordée à l'astrologie ${ }^{50}$. La description des portes du palais, qui occupe la majeure partie de notre extrait, ne peut que nous faire penser aux représentations que comportaient également les portes du temple d'Apollon Palatin, largement commentées par les poètes de l'époque ${ }^{51}$. Si le contenu de cette représentation diverge cependant nettement de celui des portes du temple palatin, c'est que, selon nous, la représentation poétique agit ici comme une réactualisation des enjeux du Principat : il ne s'agit plus de mettre en avant la valeur guerrière du Prince, en train de pacifier l'Empire, mais bien de montrer que la pax augusta et l'établissement de l'ordre qui en découle sont désormais une réalité effective ${ }^{52}$. Enfin, le titre de pater qui est attribué cinq fois à Phoébus ${ }^{53}$ nous rappelle en creux celui de Pater Patriae qu'a obtenu Auguste en 2 av. J.-C.

Apollon, s'il peut être perçu comme le porte-parole du poète à certains égards, est donc plus sûrement l'avatar mythologique d'Auguste dans le récit. Or, dans la perspective d'un rapprochement entre la figure du Prince et celle du dieu, le titre de pater qui est donné à Apollon dans la narration ovidienne est significatif, car il témoigne d'une ambiguïté fondamentale du personnage, que souligne tout particulièrement la première adresse de Phaéthon au dieu (Ov., Met. 2, 35-38) :

Ille refert: "o lux immensi publica mundi,

Phoebe pater, si das usum mihi nominis huius

Nec falsa Clymene culpam sub imagine celat,

Pignora da, genitor" [...]

«Phaéthon répond: "ô lumière commune à l'immense univers, Phoébus père, si tu me permets d'employer ce nom, et si Clyméné ne dissimule pas sa faute sous une fausse représentation, donne-moi une garantie certaine, toi, mon géniteur" " [trad. Chamonard modifiée].

21 L'interpellation pater accompagnée du nom du dieu est parfaitement classique dans le vocabulaire poétique de l'époque $\mathrm{e}^{54}$, où le substantif traduit aussi bien la déférence du locuteur que la puissance du dieu invoqué. Mais dans ces quelques vers s'opère une superposition de sens, puisqu'à ce rappel d'une dénomination formulaire s'ajoute une signification familiale qui est ici tout l'enjeu de la reconnaissance paternelle espérée par Phaéthon ${ }^{55}$. Phoébus est bien ici un pater au sens premier du terme, comme le souligne la dérivation sémantique introduite deux vers plus loin, avec la mention de genitor $^{56}$. Ainsi, Apollon, s'il a bien vocation à être l'avatar mythologique du Prince, agit 
alors comme miroir inversé : il représente tout ce que le Prince ne doit pas faire en un domaine bien spécifique, et d'une actualité prégnante au moment de la rédaction des Métamorphoses, à savoir l'épineuse question de la succession ${ }^{57}$. En effet, à cette époque, Auguste laissait de plus en plus apparaitre sa volonté d'une succession pensée sur le modèle dynastique, que la mort successive de ses deux fils adoptifs rendait problématique $^{58}$. Le Soleil est ici l'exemple inquiétant du gouvernant qui fait passer ses relations familiales en premier, aux dépens du bien-être de l'État. La mort du jeune homme lui-même, loin de rétablir le Soleil dans la fonction qui devrait être la sienne, constitue l'acmé d'une représentation divine conçue comme miroir antithétique du dirigeant idéal (Ov., Met. 2, 381-385) :

Squalidus interea genitor Phaethontis et expers

Ipse sui decoris, qualis, cum deficit orbem,

Esse solet, lucemque odit seque ipse diemque

Datque animum in luctus et luctibus adicit iram

officiumque negat mundo. [...]

«Cependant, le père de Phaéthon, décoloré, renonçant lui-même à son propre éclat, tel qu'il a coutume d'être lorsqu'il fait défaut au monde, n'a que haine pour la lumière, pour lui-même, pour le jour, et s'abandonne à son deuil, et ce deuil redouble sa colère; il refuse d'accomplir son devoir envers le monde » [trad. J. Chamonard modifiée].

Ici se trouve l'unique occurrence du génitif Phaethontis pour préciser l'appellation de genitor désignant Phoébus, lors même que la mort du jeune homme vient de nous être décrite. Il s'agirait alors d'insister sur l'obstination du Soleil à se comporter en père, bien que ne l'étant plus ${ }^{59}$. C'est bien cette propension à agir comme un particulier qui met en péril le bon fonctionnement du monde ${ }^{60}$, et que Jupiter devra ensuite sanctionner. Comme l'ont déjà suggéré certains critiques ${ }^{61}$, Phaéthon serait alors un avatar du jeune Gaius mort il y a peu ${ }^{62}$.

Ovide nous présente donc en réalité un double miroir inversé d'Auguste, où tantôt Phaéthon, tantôt Phoébus, agissent comme figures de repoussoir ${ }^{63}$. On peut en trouver un indice jusque dans la proximité phonique qui relie le nom des deux personnages, Phoébus/Phaéthon ${ }^{64}$. Mais la confrontation de ces deux miroirs nous amène à préciser la spécificité de leur fonctionnement chez Ovide: en effet, il convient de remarquer que, si Phoébus agit ici comme contre-modèle, ce n'est pas, à l'instar de Phaéthon, par manque de savoir (Ov., Met. 2, 124-125) :

Imposuitque comae radios praesagaque luctus

Pectore sollicito repetens suspiria dixit

"Phoébus dépose les rayons du soleil sur la chevelure de Phaéthon, et, tirant

encore de sa poitrine anxieuse des soupirs pleins de la prescience de son deuil, il

dit... » [trad. Chamonard modifiée]

Le narrateur ovidien prend soin de souligner qu'en plus d'avoir une connaissance parfaite de toutes les choses qui se produisent sur terre puisqu'il les voit ${ }^{65}$, il a également la prescience du sort qui attend son fils, don mantique en conformité avec les attributions traditionnelles d'Apollon ${ }^{66}$. Or, ce sont précisément ces deux vers qui introduisent les conseils de Phoébus à son fils : comme pour pallier sa propre erreur, le Soleil donne des conseils à son fils, feignant de croire qu'ils pourront être efficaces. C'est donc l'échec du dieu à représenter le roi idéal, jusque dans la gestion de sa succession, qui génère l'introduction du second miroir qui, de ce fait, échouera également à faire de Phaéthon le Prince idéal. Y aurait-il là un constat prospectif amer de la part d'Ovide sur la possibilité que son mythe puisse réussir à guider le Prince? 

aide à préciser la fonction didactique qu'Ovide confère à sa narration mythologique : face à l'échec de Phaéthon à conduire le char solaire, face à l'échec de Phoébus à agir pour résoudre les dégâts causés par son fils, Jupiter, roi suprême, intervient ${ }^{67}$. L'expression pater omnipotens par laquelle le dieu est désigné au vers 304, où pater correspond bien cette fois à la désignation traditionnelle des dieux, laisse espérer que Jupiter se comportera, lui, en bon souverain, qu'il sera capable d'agir pour le bien commun. C'est d'ailleurs bien le rôle que Tellus l'exhorte à endosser ${ }^{68}$, et qu'il réussira à assumer ${ }^{69}$. Pourtant, lui aussi échoue à représenter le roi idéal : d'abord, parce que sans l'exhortation de Tellus ${ }^{70}$, il semble que Jupiter ne serait pas intervenu pour lutter contre la catastrophe; or, la déesse, qui use de nombreux procédés oratoires, place également l'enjeu de l'action sur le terrain personnel, comme le montrent les adjectifs possessifs, voire familial. Même si l'on ne sait pas exactement ce qui, dans le long discours de la déesse, décida Jupiter à intervenir, le fait même que Tellus doive l'exhorter suggère que ses motivations ne sont pas, ou pas uniquement, d'ordre éthique. Au-delà des motivations personnelles de son action, ce sont les moyens euxmêmes par lesquels Jupiter règle la situation qui sont problématiques (Ov., Met. 2, 304-312) : contestation successive, on se rend compte, lorsqu'on lit attentivement le texte, qu'il ne s'agit en fait que de faire voir à chacun la nécessité d'agir; le conseil des dieux, topique de l'épopée, n'a plus vocation à prendre des décisions concernant les modalités d'action, mais simplement à approuver qu'il y ait action ${ }^{71}$. Or la manière dont Jupiter souhaitait résoudre le problème, une pluie abondante, échoue, et Ovide se plaît à ne pas nous en donner la raison, de sorte que la solution adoptée pour finir semble pour ainsi dire injustifiée, et provoquera la contestation ${ }^{72}$, à laquelle Jupiter répondra de manière ambiguë (Ov., Met. 2, 396-397) :

\footnotetext{
[...] missos quoque Iuppiter ignes

Excusat precibusque minas regaliter addit.

«Jupiter, de son côté, se justifie d'avoir dû lancer sa foudre et, en souverain, il accompagne ses prières de menaces » [trad. J. Chamonard].
}

27 Il semble que les justifications données par Jupiter pour avoir lancé la foudre sur Phaéthon ne suffisent pas à rendre raison à Phoébus (soit parce que ce dernier ne peut que raisonner comme un père, soit parce que Jupiter n'a pas agi comme un bon roi en 
prenant seul ses décisions), en sorte qu'il doit «ajouter des menaces aux prières ». Si l'adverbe regaliter porte bien sur le verbe addit, un problème d'interprétation se pose : est-ce d'ajouter des menaces aux prières ou bien simplement d'avoir usé de prières qui est «digne d'un roi »? Si precibus n'est qu'une formule résomptive de la première proposition principale, interprétation à privilégier puisque d'autres prières n'ont pas été mentionnées, alors c'est bien la première hypothèse qui l'emporte. Agir comme un roi, c'est nécessairement agir comme un personnage omnipotens et user de menaces. Aussi Ovide suggérerait-il que sa narration mythologique, si elle a vocation à guider Auguste, ne peut obtenir les effets désirés, car c'est la fonction de roi, dans ses fondements mêmes (son pouvoir décisionnaire, sa gestion de la succession ${ }^{73}$ et les prétentions à l'apothéose qu'elle semble légitimer), qui est, non pas nécessairement condamnable ${ }^{74}$, mais problématique.

Si le terme de "miroir du Prince» est anachronique pour qualifier la narration ovidienne, force est de constater que l'ensemble des procédés qui seront formalisés ultérieurement, au moment de la création du genre médiéval, sont déjà mis à profit par Ovide, et que leur analyse nous aide à appréhender les enjeux de sa réflexion politique. Dans cette perspective, le mythe de Phaéthon, par la richesse de ses lectures allégoriques, nous présente donc bien ce que l'on pourrait qualifier de triple miroir du Prince. Faisant miroiter, grâce au système analogique, les reflets d'un éloge mais aussi d'une critique ${ }^{75} \mathrm{du}$ régime augustéen, Ovide use de sa narration pour délivrer un enseignement non pas politico-éthique, mais bien plutôt philosophico-éthique, en laissant volontairement le lecteur dans l'aporie sur l'utilité et le sens du mythe. Si Phaéthon est bien l'agent direct de l'ekpurosis du monde, sa qualité de mortel, même à demi divin, ne lui permettait pas d'avoir de telles conséquences cosmiques sans les erreurs commises par les deux figures traditionnellement exemplaires que sont Phoébus et Jupiter. Comment donc agir en tant que mortel, en tant que Prince aux prétentions divines, ou bien même en tant que roi des dieux?

Le miroir du Prince est donc ici un triple échec. Est-ce à dire que le poète ne se fait aucune illusion quant à la possibilité d'éduquer un Prince déjà âgé, et dont le régime se montre de plus en plus dirigiste et autoritaire? Peut-être, mais nous préférons pour notre part la lecture optimiste: il convient de remarquer que deux des trois personnages ici mis en scène, les rois divins Phoébus et Jupiter, furent volontairement associés à la figure du Prince dans la propagande augustéenne ${ }^{76}$ afin de véhiculer une brillante image de son propre règne. Ovide prendrait ici le Prince à son propre piège : à trop user de symboles, Auguste se livrait lui-même à l'arbitraire du signe et aux dangers de l'interprétation d'une représentation dont la signification ultime ne peut dépendre que du destinataire ${ }^{77}$. Il s'agirait également de dire l'impossibilité, pour un homme d'État, à s'associer à la théologie mythique et à son immoralité inhérente, sous peine d'un grave échec. Le triple miroir du Prince proprement dit échoue à instruire Auguste, parce que la littérature n'est pas là pour instruire le Prince sur la manière d'être un bon gouvernant. En revanche, elle est là pour lui demander : «Es-tu sûr de bien gouverner? Regarde les mauvais exemples auxquels tu peux être comparé et les conséquences que tes actions risquent d'entraîner. Ne peux-tu trouver une meilleure solution? » Le mythe de Phaéthon, bien intégré en cela dans une réflexion propre aux Métamorphoses d'Ovide, pose plus de questions qu'il n'en résout. Il y aurait donc bien une leçon didactique du mythe ovidien, mais pas celle attendue. 


\section{BIBLIOGRAPHIE}

BASS R. C. 1977, « Some Aspects of the Structure of the Phaethon Episode in Ovid's Metamorphoses ", CQ 27, p. 402-408.

BRISSON J.-P. 1992, Rome et l'âge d'or. De Catulle à Ovide, vie et mort d'un mythe, Textes à l'appuis. Histoire classique, Paris, Éd. La Découverte.

CUSSET C. 2009, « Irrévérence de l'éloge du pouvoir lagide dans les Idylles de Théocrite », in B. DELIGNON, Y. ROMAN (dir.), Le poète irrévérencieux, Coll. du CEROR 32, Lyon, Université Jean Moulin Lyon 3, CERGR, p. 89-102.

DEREMETZ A. 1995, Le miroir des muses. Poétiques de la réflexivité à Rome, Racines \& modèles, Villeneuve-d'Ascq, Presses universitaires du Septentrion.

DETIENNE M. 1992, L'invention de la mythologie, Coll. Tel 212, Paris, Gallimard.

JóNSSON E. M. 1995, Le miroir. Naissance d'un genre littéraire, Histoire, Paris, Les Belles Lettres.

FABRE-SERRIS J. 1995, Mythe et poésie dans les Métamorphoses d'Ovide. Fonctions et significations de la mythologie dans la Rome augustéenne, Études et commentaires 104, Paris, Klincksieck.

FELDHERR A. 2016, « Nothing like the Sun: Repetition and Representation in Ovid's Phaethon Narrative ", in L. FULKERSON, T. STOVER (éd.), Repeat Performances. Ovidian Repetition and the Metamorphoses, Wisconsin Studies in Classics, Madison, The University of Wisconsin Press. FONTENROSE J. 1940, «Apollo and the Sun-God in Ovid », TAPhA 61, 4, p. 429-444. DOI : 10.2307/291381. URL : https://www.jstor.org/stable/i212995.

GAGÉ J. 1955, Apollon romain. Essai sur le culte d'Apollon et le développement du ritus graecus à Rome des origines à Auguste, Bibliothèque des Écoles françaises d'Athènes et de Rome 182, Paris, E. de Boccard.

GLARE P. G. W., UNIVERSITY OF OXFORD (dir.) 1968, Oxford Latin Dictionary, Oxford, Clarendon Press, 2 vol.

HOLLARD V. 2016, «La fonction politique du poète dans la cité à l'époque d'Auguste : l'exemple d'Horace (Odes et Carmen saeculare) », in B. DELIGNON, N. LE MEUR, O. THÉVENAZ (éd.), La poésie lyrique dans la cité antique. Les Odes d'Horace au miroir de la lyrique grecque archaïque. Actes du colloque organisé les 6-8 juin 2012 par l'ENS de Lyon, Hisoma, UMR 5189 et l'Université de Lausanne, Coll. du CEROR 19, Lyon, CEROR, p. 49-62.

LAIGNOUX R. 2011, « L'utilisation de la religion dans la légitimation du pouvoir : quelques pistes de recherche pour les années 44-42 av. J.-C. », Cahiers " Mondes anciens » 2. DOI : 10.4000/ mondesanciens.360. URL : http://journals.openedition.org/mondesanciens/360.

LEDENTU M. 2012, In arto labor ? L'écriture et le pouvoir sous le Principat d'Auguste. Enjeux et modalités d'une interaction, Mémoire inédit présenté en Sorbonne en décembre 2012 pour l'Habilitation à diriger des recherches.

MILLER J. F. 2009, Apollo, Augustus, and the Poets, New York, Cambridge University Press.

NAEGLE G. 2010, «Resveillier ceulx qui dorment en pechié : Philippe de Mézières et la tradition des miroirs du prince », Le Moyen Âge 106, p. 625-643. DOI : 10.3917/rma.163.0625. URL : https:// www.cairn.info/revue-le-moyen-age-2010-3-page-625.htm. 
OTIS B. 1975, Ovid as an Epic Poet, 2nd ed., Cambridge, Cambridge University Press.

POULLE B. 2002, « Phaéthon et la légitimité d'Auguste », in M. FARTZOFF, E. SMADJA, E. GENY (éd.), Pouvoir des hommes, signes des dieux dans le monde antique, ISTA 852, Besançon, Presses universitaires franc-comtoises, p. 125-134. URL : www.persee.fr/doc/ ista_0000-0000_2002_act_852_1_1653.

RUDHARDT J. 1997, « Le mythe de Phaéthon », Kernos 10, p. 83-95.

SCHMITZER U. 1990, Zeitgeschichte in Ovids Metamorphosen. Mythologische Dichtung unter politischem Anspruch, Beiträge zur Altertumskunde 4, Stuttgart, Teubner.

VIDEAU A. 2010, La poétique d'Ovide, de l'élégie à l'épopée des Métamorphoses. Essai sur un style dans l'histoire, Rome et ses renaissances, Paris, PUPS.

\section{NOTES}

1. Voir la première partie de l'Introduction de ce numéro d'Interférences.

2. Cette réflexion a été particulièrement valorisée au milieu des années quatre-vingtdix : voir en particulier DEREMETZ 1995 et JóNSSON 1995, p. 76 : « Il n'y a pas de relation univoque et nécessaire entre le niveau lexical et sémantique et celui des concepts, idées et sentiments. Une idée, par exemple, peut apparaître et exister sans qu'il y ait un mot précis pour la désigner ».

3. U. SCHMITZER (1990) avait déjà osé ce qui pourrait sembler être la même entreprise. Néanmoins, en dépit d'un titre extrêmement similaire au nôtre, sa réflexion est très différente, quoique complémentaire à bien des égards, de celle proposée ici. En effet, son article consiste davantage à recenser les points de contact élaborés par Ovide dans sa narration entre le mythe de Phaéthon et l'histoire d'Auguste (utilisant ainsi le terme de «miroir » au sens de reflet à l'identique) qu'à considérer le «miroir » dans un sens technique, en tant que discours pédagogique adressé au Prince. Ses analyses de détail, en outre, ne recoupent que rarement les nôtres; sa réflexion porte sur quatre passages en particulier : l'ekphrasis du palais, le zodiaque sur la route de Phaéton, le catalogue des montagnes et le catalogue des eaux.

4. Malgré les dissensions qui sont apparues pour établir les bornes du texte, nous adoptons ici la délimitation généralement admise, et à laquelle réfléchit R. C. BASS (1977). Le mythe commence au vers 747 du premier livre des Métamorphoses, pour s'achever au vers 339 du deuxième livre.

5. Concernant la question longuement débattue de l'immoralité et de la fausseté de la matière mythologique, voir la condamnation virulente de Xénophane, et la position plus ambiguë de Platon, qu'analyse notamment M. Detienne (DETIENNE 1992, chap. 5, "La cité défendue par ses mythologues ", p. 155-189).

6. L'importance des jugements émis par le narrateur omniscient pour la compréhension des mythes et de leur morale dans les Métamorphoses a notamment été soulignée par VIDEAU 2010, p. 480-481 (à propos des caelestia crimina représentés par Arachné sur sa toile).

7. Voir la deuxième partie de l'Introduction de ce numéro d'Interférences; le miroir est une sous-catégorie du genre didactique visant à édifier moralement son lecteur, et construit le reflet idéal du destinataire visé. 
8. Ov., Met. 2, 50-102.

9. Ov., Met. 2, 126-149.

10. Ov., Met. 2, 103 et 126.

11. On peut aussi remarquer que le Soleil introduit son propos en disant dissuadere licet (Ov., Met. 2, 53), ce qui, par l'étymologie même du verbe à l'infinitif (dis-suadere), implique bien l'idée d'un conseil négatif.

12. Ov., Met. 2, 146.

13. Sauf indication contraire, nous reprenons dans cet article la traduction de J. Chamonard aux éditions Garnier, que nous modifions occasionnellement.

14. Les injonctions de Phoébus à son fils se retrouvent, assez logiquement, en conclusion de son discours ; voir notamment Ov., Met. 2, 89-102.

15. Voir par eX. SCHMITZER 1990 ou POULLE 2002.

16. VIDEAU 2010, p. 529 : «Lue sous l'angle politique, l'expression currum tenere ne peut que rappeler la métaphore usitée du 'char de l'État'. Aussi Phaéton n'a pu ni su currum tenere: c'est-à-dire 'retenir', 'freiner', mais aussi 'posséder', 'conserver' ce char, emblème du pouvoir ». A. DEREMETZ (1995, chap. 5) rappelle également que «la course du char et le périple du navire sont précisément, depuis toujours et dans tous les genres, les métaphores les plus utilisées par les poètes pour représenter la composition $\mathrm{du}$ poème ». Cette superposition de sens est particulièrement intéressante dans la perspective d'un miroir du Prince : seul le poète, en maîtrisant bien son propre navire ou char, son poème, peut rendre le dirigeant à même de bien conduire le sien, l'État.

17. Voir en particulier Ov., Met. 2, 161-167: Sed leue pondus erat nec quod cognoscere possent / Solis equi solitaque iugum grauitate carebat;/ Vtque labant curuae iusto sine pondere naues / Perque mare instabilis nimia leuitate feruntur, / Sic onere adsueto uacuus dat in aere saltus / Succutiturque alte similisque est currus inani, « Mais la charge était légère, telle que les chevaux du Soleil ne pouvaient la reconnaître, et le joug ne pesait pas de la lourdeur accoutumée. Et, de même que les navires aux flancs courbes, dépourvus du lest nécessaire, dansent sur les flots et sont emportés à travers la mer, instables parce que trop légers, ainsi le char allégé de son poids habituel bondit dans les airs, ballotté de haut en bas, comme un char vide ».

18. Voir notamment GLARE, UNIVERSITY OF OXFORD 1968, s.v. « grauis ».

19. Ov., Met. 2, 1 : Regia Solis.

20. Ov., Met. 2, 5-7: [...] nam Mulciber illic / Aequora caelarat medias cingentia terras / Terrarumque orbem caelumque, quod imminet orbi, « Mais l'art l'emportait encore sur la matière. Car Mulciber y avait ciselé les eaux entourant les terres placées au milieu, l'orbe du monde et le ciel, suspendu au-dessus du monde »[trad. J. Chamonard modifiée].

21. Ov., Met. 2, 1-4: Regia Solis erat sublimibus alta columnis, / Clara micante auro flammasque imitante pyropo; / Cuius ebur nitidum fastigia summa tegebat, / Argenti bifores radiabant lumine ualuae, «Le palais du Soleil s'élevait porté par de hautes colonnes, tout brillant du scintillement de l'or et du pyrope imitant les flammes; l'ivoire luisant en recouvrait, tout en haut, le faîte; les doubles battants des portes rayonnaient de l'éclat de l'argent » [trad. J. Chamonard modifiée].

22. On trouve, au vers 25 , un rythme ternaire, auquel vient s'ajouter un quatrième élément, mais dans le vers qui suit, pour ne pas rompre l'harmonie du premier groupe, 
créant ainsi un rythme binaire entre Saecula et Horae placés aux extrémités du vers. Puis, les saisons sont mentionnées une à une, chacune dans son propre vers; enfin la polysémie de aequalibus suggère bien l'idée d'une entente parfaite.

23. Voir en particulier Pl., R. 6, 488-489 b.

24. Ov., Met. 2, 59-60: "Non tamen ignifero quisquam consistere in axe / Me ualet excepto ;" [...], «"Aucun cependant n'est capable de se tenir sur le char qui porte le feu, moi seul excepté" ».

25. Ov., Met. 2, 102, le Soleil achève son discours à Phaéthon en lui disant: "Sed tu sapientius opta", " "Mais toi, fais un choix plus sage" » [trad. personnelle].

26. Voir Ov., Met. 2, 58 (nescius), 148 (inscius), 170 (nec scit qua sit iter, avec allitération et assonance pour mettre l'idée en valeur), 191 (quidque agat ignarus stupet), 192 (nec nomina nouit equorum, avec polysyndète et rythme ternaire pour créer un renforcement), et 233-234 (Quoque eat, aut ubi sit, picea caligine tectus / Nescit, avec enjambement du verbe nescio pour souligner le renforcement).

27. Voir Ov., Met. 2, 32 (iuuenem), 55 (tam puerilibus annis), 106 (iuuenem), 127 (puer), 150 (iuuenali corpore), 198 (puer). Il est significatif de constater que la liste des termes insistant sur sa jeunesse, comme de ceux insistant sur son inexpérience, s'achève avec la description des conséquences qu'a eues la course agitée de Phaéthon. Sa chute tragique, tout en mettant fin à sa vie, termine l'apprentissage difficile qu'il vient de réaliser: fort de son expérience nouvelle, il a appris qu'être roi n'était ni une tâche facile, ni une tâche enviable.

28. Voir en particulier Ov., Met. 2, 126-127 : "Si potes his saltem monitis parere parentis, / Parce, puer, stimulis et fortius utere loris", " "Si tu peux du moins obéir à mes recommandations paternelles, n'use pas, mon enfant, de l'aiguillon, et tiens plutôt d'une main forte les rênes" ".

29. Ov., Met. 2, 42-43 : [...] "nec tu meus esse negari / Dignus es et Clymene ueros" ait "edidit ortus", " "Mais toi, tu ne mérites certes pas que je te renie pour mon fils", dit-il, "et Clyméné t’a fait connaître tes véritables origines" » [trad. J. Chamonard modifiée].

30. Ov., Met. 2, 31-32: Inde loco medius rerum nouitate pauentem / Sol oculis iuuenem, quibus adspicit omnia, uidit, "Alors, placé au milieu d'eux, le Soleil, de ses yeux qui voient tout, aperçut le jeune homme, effrayé par la nouveauté du spectacle ».

31. Ce procédé est peut-être à mettre en lien avec le contrôle qu'Auguste exerçait sur la littérature à la fin de son règne. Sans pour autant parler d'un moyen de contourner la censure, il s'agirait simplement de ne pas prodiguer trop directement ses conseils au Prince, ce qui semblerait inconvenant de la part d'un poète.

32. Voir NAEGLE 2010, en particulier p. 633 : « Pour rendre l'expression de leur critique plus acceptable, la plupart des traités de réforme utilisent quelques astuces littéraires typiques. [...] Le songe est une forme ambiguë qui permet de garder une certaine distance par rapport au contenu de la critique exprimée ».

33. Pour la théologie solaire qui se développe autour du dieu Apollon au moment du Principat d'Auguste, voir GAGÉ 1955 ( $3^{\mathrm{e}}$ partie « Regnum Apollinis », chap. 6 « Les destins de l'Apollon impérial ; vers une théologie solaire», p. 639-684). La question de savoir si Ovide fait dans ses œuvres l'assimilation entre le Soleil et Phoébus est complexe et a été l'objet de nombreuses controverses. J. FONTENROSE (1940) affirme que les deux seuls points de convergence réels sont leur parenté avec Diane-Phoébé, et leur appellation commune, Phoebus, mais que les deux dieux sont considérés comme deux entités 
distinctes. Cette hypothèse a depuis emporté l'adhésion, et nous la suivons. Cependant, nous voudrions ajouter, avec U. Schmitzer (1990, p. 89-90), que, même si Apollon et Sol ne sont jamais confondus chez Ovide, l'assimilation, initiée par Auguste avec la représentation solaire de l'Apollon palatin, reste opérante ici. L'alternative fait partie, selon nous, des nombreux éléments qui permettent différents niveaux de lecture du mythe.

34. Ov., Met.1, 751-753: [...] Quem quondam magna loquentem/ Nec sibi cedentem Phoeboque parente superbum / Non tulit Inachides [...], « Comme un jour, plein de jactance, Phaéton, refusait de céder [à Epaphus], tout fier de son père Phoébus, le petit-fils d'Inachus ne le supporta pas » [trad. J. Chamonard modifiée].

35. U. SCHMITZER (1990, p. 90) note cependant qu'il existe une parenté très forte entre Phaéton, fils du Soleil, qui vit avec Mérops et sa mère, et Auguste, fils d'Apollon, qui vit avec sa mère Atia et Octavius. C'est sans doute le motif de la jeunesse du personnage qui permet ce parallèle.

36. Ov., Met. 2, 37-38: "Pignora da, genitor, per quae tua uera propago / Credar et hunc animis errorem detrahe nostris", « "Donne-moi un gage, toi qui m'as engendré, grâce à quoi nul ne doute que je sois de ta lignée, et délivre mon esprit de cette incertitude" ». Le jeune Octavien quant à lui, avant même la bataille d'Actium, qui vient confirmer le protectorat qu'exerce Apollon sur le futur Prince, revendiquait un lien personnel et privé avec le dieu, lorsqu'il décida, en $36 \mathrm{av}$.J.-C., de faire construire un temple à Apollon sur le domaine de sa maison.

37. Voir notamment Ov., Met. 15, 845-851.

38. Nous savons qu'Ovide commença à composer les Métamorphoses en 2 av. J.-C., et en termina la rédaction en 8 apr. J.-C. Si nous ne pouvons savoir à quel moment précis il écrivit spécifiquement le mythe de Phaéthon, nous pouvons tout à fait supposer que, jusqu'à la publication du recueil, il gardait la possibilité de remanier son œuvre pour y aborder les questions essentielles de la plus récente actualité. La préparation de la future divinisation du Prince était, quoi qu'il en soit, officielle de longue date: les Augustalia de 19 av. J.-C. furent une étape franchie, puisque «c'était la première fois qu'un festival était consacré à une personne vivante » et que ces fêtes «n'impliquaient ni plus ni moins qu'un culte rendu à la personne d'Auguste » (LEDENTU 2012, p. 156-157) ; les Jeux Séculaires de 17 av. J.-C. ont achevé de le présenter comme la figure salvatrice de son peuple, sur laquelle veillaient tous les dieux et en particulier Apollon et Diane; en 7 av. J.-C., avec la réforme des Compitalia, Auguste associe son Genius au culte des Lares (LEDENTU 2012, p. 291), avant de parachever cette réforme, en 5 av. J.-C., où apparaît le culte du numen Augusti (HOLLARD 2016, p. 60).

39. Quelques décennies plus tard, Vespasien se moque d'une divinisation qui est désormais devenue automatique, mais qui ne peut survenir qu'après la mort du Prince lorsque, juste avant d'expirer lui-même, il aurait affirmé : «ah, je crois que je deviens dieu » (voir Suet., Ves. 23, 8 : "Vae", inquit, "puto, deus fio").

40. Ovide traduit ce danger, en termes mythologiques, par le risque de retomber dans le chaos originel : voir les propos de Tellus à Jupiter, Ov., Met. 2, 298-299: "Si freta, si terrae pereunt, si regia caeli, / In chaos antiquum confundimur". [...], " "Si les mers, si la terre, si la royale demeure du ciel périssent, nous retombons dans la confusion de l'antique chaos" ". 
41. Ov., Met. 2, 327-328 : "Hic situs est Phaethon currus auriga paterni ; / Quem si non tenuit, magnis tamen excidit ausis.", « "Ci-git Phaéthon, qui conduisit le char paternel. S’il ne put le diriger, du moins périt-il victime d'une noble audace" ".

42. Voir notamment RUDHARDT 1997, p. 93.

43. Voir Ov., Met. 2, 88-89: At tu, funesti ne sim tibi muneris auctor, / Nate, caue, dum resque sinit, tua corrige uota, «mais toi pour que je ne sois pas pour toi l'auteur d'un don funeste, mon fils, prends garde tant que cela peut se faire, fais une voeu meilleur », et 137 : medio tutissimus ibis, « au milieu ta course sera parfaitement sûre ».

44. Ov., Met. 2, 91-92: "Pignora certa petis? do pignora certa timendo / Et patrio pater esse metu probor": " "Tu demandes un gage certain? Eh bien! ce gage certain, mes craintes te le donnent et mes alarmes paternelles sont la preuve que je suis ton père" ".

45. Pour la tension qui se crée entre le Prince et les poètes à l'époque augustéenne autour de la revendication du patronage d'Apollon, voir en particulier MILLER 2009.

46. Voir supra note 33.

47. U. SCHMitZeR (1990, p. 91) a fait le rapprochement entre cette description du palais du Soleil et la description du temple d'Apollon Palatin chez Properce (Prop., 2, 31, 1-12). Il ajoute, p. 92, que le mot regia, en ouverture de livre, renvoie au siège du Grand Pontife qu'Auguste a transporté sur le Palatin.

48. Ov., Met. 2, 107-108: Aureus axis erat, temo aureus, aurea summae / Curuatura rotae, radiorum argenteus ordo, "D'or était l'essieu, le timon d'or, d'or la jante courbe entourant la roue, d'argent la série des rayons ».

49. Pour l'actualité politique du mythe de l'âge d'or et les manipulations poétiques subies par le mythe à l'époque d'Auguste, notamment chez Ovide, voir BRISSON 1992, en particulier chap. 11, p. 175-186, qui fait des Métamorphoses, peut-être de manière un peu trop systématique cependant, une dénonciation farouche des prétentions du régime à endosser le titre de nouvel âge d'or. J.-P. Brisson note que, curieusement, c'est « quand le mythe fut invoqué pour servir de modèle à une souveraineté inavouée (le Principat), [...] qu'il s'est vidé de sa substance»(p.9). La réduction de l'âge d'or, dans la description ovidienne, à la seule présence matérielle de l'or, qui contraste avec la catastrophe sur le point de se produire, l'ekpurosis, est peut-être une manière pour Ovide de suggérer que l'appellation saeculum aureum pour désigner le Principat est bien dépourvue de toute consistance.

50. A. VIDEAU (2010, p. 521) analyse la figure du Soleil telle qu'elle est représentée au début du livre 2 des Métamorphoses, au sein du mythe de Phaéthon, et démontre qu'elle peut être assimilée à Auguste, notamment parce que «le dieu est [...] représenté en maitre du Temps : maître de la régularité parfaite de son ordre cosmique ». Selon elle, "une telle représentation ne peut qu'évoquer le rôle joué par Auguste dans l'achèvement du calendrier de César ».

51. Voir notamment Hor., Carm. 4, 6 ; Prop., 2, 31 ; et la réinterprétation poétique qu'en fait Virgile, dans Verg., A. 6, 20-33 et dans Verg., G. 3, 26-39.

52. Voir en particulier Ov., Met. 2, 6-7, qui traduit cette idée en termes mythologiques, en opposant l'ordre du monde au chaos initial décrit au livre 1 des Métamorphoses.

53. Ov., Met. 2, 36, 49, 92, 122 et 329. On trouve une sixième occurrence au vers 304, mais qui qualifie Jupiter (pater omnipotens). 
54. Voir par ex. Verg., A. 1, 60 (pater omnipotens) ; 8, 357 (Ianus pater) ; G. 2, 4 et 7 (pater o Lenaee) ; Prop., 3, 4, 11 (Mars pater) ; Ov., Met. 13, 669 (Bacche pater).

55. L'idée de puissance afférente à l'adjonction du substantif pater au nom du dieu dans les invocations tient au fait que le suppliant confère alors à la divinité le même pouvoir sur lui que celui possédé par le paterfamilias sur sa famille, jusqu'au droit de vie et de mort. C'est cette dérivation sémantique qui permet à Phaéthon de jouer sur l'ambiguïté de sens.

56. Ov., Met. 2, 38, 40, 105 et 381.

57. U. SCHMitzer (1990) note également la présence de ce thème dans le mythe de Phaéthon, lorsque, dans son analyse du catalogue des montagnes mises en péril par Phaéthon, il affirme (p. 95) que le Taurus cilicien rappelle le roi-client Archélaüs qui a joué un rôle certain dans la politique intérieure romaine, en particulier autour des questions de possible succession d'Auguste. Il conclut son analyse (p. 99) en affirmant que la route de Phaéthon est une reprise symbolique de la route d'Auguste vers le pouvoir, puis au pouvoir.

58. En 17 av. J.-C., les Jeux Séculaires avaient étroitement lié le sort de la Ville à celui de son chef, qui était désormais perçu comme le successeur en droite lignée d'Énée. Ce fut le premier jalon clairement dynastique du principat augustéen. En 9 av. J.-C., des fêtes en l'honneur d'Auguste et de la famille impériale entreprennent de lier Auguste non plus à son ascendance mais bien à sa descendance. En 2 av. J.-C., la dédicace de l'Ara Pacis intégrait dans l'iconographie collective le souvenir de la filiation divine de la gens Iulia. La mort de Lucius en 2 apr. J.-C., puis celle de Caius en 4 apr. J.-C., qui eut pour conséquence l'adoption de Tibère, réactualisaient une problématique qui était déjà au cœur des préoccupations du Prince depuis au moins 10 ans déjà.

59. B. OTıs (1975, p. 110) souligne, en étudiant la figure de Phoébus dans ce mythe, « his paternal and his erotic feelings $»$.

60. U. SCHMitzer (1990, p. 105) fait le parallèle avec Verg., G. 1, 466-468, où le Soleil cache sa face au monde lors de la mort de César, confirmant ainsi la lecture allégorique qu'il faut faire de ce mythe au sujet de la politique contemporaine.

61. Voir en particulier VIDEAU 2010, p. 527-535.

62. Voir VIDEAU 2010, p. 527-535. L'analogie n'est cependant pas parfaite, car nous ne pensons pas qu'il s'agisse pour Ovide de sous-entendre qu'Auguste aurait une responsabilité dans la mort du jeune homme comme Phoébus en a une dans celle de Phaéthon. L'accent est alors mis sur la figure de Phoébus comme contre-modèle pour le Prince, et non sur une analogie parfaite entre les deux jeunes hommes.

63. A. FELDHERR (2016, p. 27) note que Phaéthon a été associé tantôt à Auguste, tantôt à Gaius. Il affirme que les différentes hypothèses sont toutes convaincantes, et qu'elles ne s'excluent pas forcément; il nous semble que c'est le concept de miroir du Prince, avec ses enjeux didactiques, qui nous permet de comprendre qu'il ne faut pas faire de toutes les identifications possibles des interprétations exclusives mais bien au contraire complémentaires.

64. Phoébus lui-même, après avoir entendu le souhait de Phaéthon, affirme, en Ov., Met. 2, 50-51: "temeraria" dixit / "Vox mea facta tua est", " "C'est la témérité de mon propos", dit-il, "qui a provoqué la tienne" " [trad. personnelle]. Il suggère ainsi la correspondance qui doit s'établir entre les deux figures, dont le défaut majeur serait la témérité. 
65. Ov., Met. 2, 32: Sol oculis iunenem, quibus adspicit omnia, uidit, « le Soleil, de ses yeux qui voient tout, aperçut le jeune homme».

66. La seule ignorance qu'admet le Soleil est celle des eaux du Styx : Ov., Met. 2, 45-46: “ [...] promissis testis adesto / Dis iuranda palus, oculis incognita nostris", " "Que soit témoin de ma promesse le marais par lequel jurent les dieux, et que nos yeux ne connaissent pas" » [trad. J. Chamonard modifiée]. Or, il nous semble qu'Ovide joue ici de l'opposition traditionnelle entre divinités infernales et divinités célestes, les deux catégories ne devant jamais entrer dans la demeure l'une de l'autre, pour en fait souligner par anticipation que c'est cette ignorance, sur laquelle il fonde sa promesse, qui sera la cause de la perte de Phaéthon.

67. Nous le voyons agir à partir du vers 304 .

68. Ov., Met. 2, 299-300: “[...] eripe flammis / Siquid adhuc superest et rerum consule summae", "Soustrais aux flammes ce qui peut encore subsister et veille à sauver l'univers" ».

69. Ov., Met. 2, 312-313 : misit in aurigam pariterque animaque rotisque / expulit et saevis conpescuit ignibus ignes.

70. Ov., Met. 2, 290-294: Sed tamen exitium fac me meruisse; quid undae, / Quid meruit frater? cur illi tradita sorte / Aequora decrescunt et ab aethere longius absunt? / Quodsi nec fratris nec te mea gratia tangit, / At caeli miserere tui, « Mais suppose que j'aie mérité de périr; en quoi les ondes, en quoi ton frère l'ont-ils mérité ? Pourquoi le niveau des mers, dont le sort lui a donné l'empire, décroît-il ? Pourquoi leur distance de l'éther augmente-t-elle? Que si tu ne t'attendris pas en faveur ni de ton frère ni de moi, du moins prends pitié de ton ciel » [trad. J. Chamonard modifiée].

71. B. отіs (1975, p. 115), allant plus loin que nous ne le ferions, explique qu'avant d'agir, Jupiter fait une «brève explication, totalement dépourvue de nécessité, de son but».

72. Voir en particulier les vers Ov., Met. 2, où la voix poétique retranscrit l'épitaphe que ses sœurs offrent à Phaéthon. À propos du deuil des Héliades à la mort de Phaéthon, J. FABRE-SERRIS (1995, p. 67) affirme que "la commisération des divinités inférieures à l'égard des hommes qui ont voulu se mesurer aux dieux sera une constante dans les Métamorphoses ». C'est souligner que les divinités mineures sont une figure constante d'opposition aux dieux olympiens.

73. Voir U. SCHMITZER (1990, p. 103-104) qui affirme que l'introduction du Scorpion dans le parcours céleste de Phaéthon est une invention d'Ovide, afin de suggérer que l'étoile qui faisait à Auguste une place dans le ciel devient celle qui perd Phaéton. Or c'est l'étoile sous laquelle est né Tibère, qui est alors le successeur désigné d'Auguste. U. Schmitzer y voit une attaque violente contre Tibère, accusé à demi-mots de précipiter dans le désastre le règne d'Auguste.

74. U. SCHMITZER (1990, p. 106) conclut son article en affirmant que la prétention à une naissance divine entraîne une hybris catastrophique, que le poète représente comme une catastrophe générale ressemblant à un bilan du Principat d'Auguste. Il faut néanmoins nuancer cette affirmation: selon nous, ce n'est pas la gestion de l'Empire durant le Principat qu'Ovide interroge dans ce mythe, mais bien la manière dont Auguste entend gérer la postérité de son œuvre. Plus encore, nous pensons qu'Ovide, loin de donner une conclusion univoque à son mythe, n'impose pas à son lecteur un constat aussi négatif et radical concernant le Principat. 
75. En cela, Ovide n'innove pas. C. CUSSET $(2009$, p. 93) conclut ainsi son analyse des analogies mythologiques présentes dans les Idylles de Théocrite: "Théocrite semble bien [...] vouloir jouer en tous les sens des effets incertains d'une surimpression des données mythologiques à la réalité historique contemporaine. On voit se mettre en place une sorte d'équivalence instable entre Héraclès, Alexandre, Lagos, Ptolémée Sôter et Ptolémée Philadelphe d'une part, Héra, Olympias, Arsinoé d'autre part, en fonction de l'angle selon lequel on envisage le récit de l'épisode. Cette ambiguïté volontaire permet d'associer, au sein du même récit, les modes antithétiques de l'éloge et de l'irrévérence ».

76. En cela, Auguste, durant le Principat, n'innove pas: voir LAIGNOuX 2011, p.4, qui rappelle que, en particulier à l'époque des guerres civiles, l'un des trois procédés religieux de légitimation de son pouvoir était bien déjà la mise en évidence de liens privilégiés avec les divinités.

77. Ovide joue donc du rôle herméneutique confié au lecteur, sur lequel repose nécessairement la signification du mythe, compte tenu de la grande diversité symbolique impliquée par la métaphore catroptique (voir l'Introduction de ce numéro d'Interférences).

\section{RÉSUMÉS}

En soulignant dès les premiers vers l'omniprésence du symbolisme augustéen, Ovide fait du mythe de Phaéthon le reflet du Principat, non pas tant pour ses réussites - comme on pourrait s'y attendre dans un mythe qui fait du monde divin une transposition du régime augustéen -, mais pour les échecs qu'il risque d'encourir. La réflexion menée sur la manière dont doit être gérée la succession d'un être tout-puissant fait tour à tour de Phébus, de Phaéthon et de Jupiter les contre-modèles présentés au Prince. Le mythe ovidien agit donc comme miroir inversé, et, dans un traitement du mythe propre à la poétique ovidienne, souligne les problèmes sans fournir de réponse, laissant celle-ci à l'entière sagacité du lecteur.

By pointing out straight from the beginning the overwhelming presence of Augustan symbolism, Ovid constructs the myth of Phaethon as a reflect of the Principate, not as much for his successes -as we can expect for a divine transposition of the Augustan regime-as for the failures incurred. Ovid questions the way the succession of an almighty person must be handled by presenting successively Phoebus, Phaethon and Jupiter as countermodels to Augustus. As such, the ovidian myth is a reverse mirror, and, in a typical process of the ovidian poetics, it enhances issues without offering solutions, which must be found by the sagacious reader. 
INDEX

nomsmotscles Platon, Ovide, Properce, Sénèque, Suétone, Virgile

Mots-clés : Auguste, Principat, succession dynastique, mythe, représentation du pouvoir à Rome, âge d'or, poésie didactique

Keywords : August, Principate, dynastic succession, myth, Roman representations of power, Golden Age, didactic poetry

\section{AUTEURS}

\section{ALIÉNOR CARTOUX}

Université Jean Moulin Lyon 3, UMR 5189 HiSoMA 